\title{
Operation of THz p-Ge laser in a closed cycle refrigerator
}

\author{
C.J. Fredricksen ${ }^{1}$, A.V. Muravjov², and R.E. Peale 2 \\ 1Zaubertek, Inc., 1809 E. Broadway St. \#313, Oviedo, FL \\ 2Department of Physics, University of Central Florida. Orlando, FL
}

\begin{abstract}
We report on development of a turn-key, cryogen-free bulk p-Ge laser which is broadly tunable over the range 1.5 to 4.2 THz. A $4 \mathrm{~K}$ closed cycle refrigerator was used to eliminate the need for liquid cryogen. A SmCo permanent magnet assembly provides the necessary magnetic field for the laser. A customized high voltage (HV) power supply and thyratron pulser were developed to replace the stack of general electronics previously used to operate the laser.
\end{abstract}

\section{INTRODUCTION}

The p-Ge laser is tunable throughout the 1.5 to $4.2 \mathrm{THz}$ region with watt-level peak output power [1]. Fig. 1 presents a characteristic laser spectrum when intracavity wavelength selection is used [2]. The laser mechanism is based on population inversion between light- and heavy-hole valence sub-bands in crossed electric and magnetic fields at cryogenic temperatures. Immersion in liquid helium has been the most convenient means of cooling the active crystal because it also provides cooling for a superconducting solenoid that gives homogeneous, tunable magnetic fields of at least $0.5 \mathrm{~T}$. However, $\mathrm{p}-\mathrm{Ge}$ lasers have also been demonstrated using permanent magnet assemblies [3, 4] in closed cycle refrigerators [5]. The purpose of this paper is to present new developments of a turn-key p-Ge laser that operates in a closed cycle refrigerator.

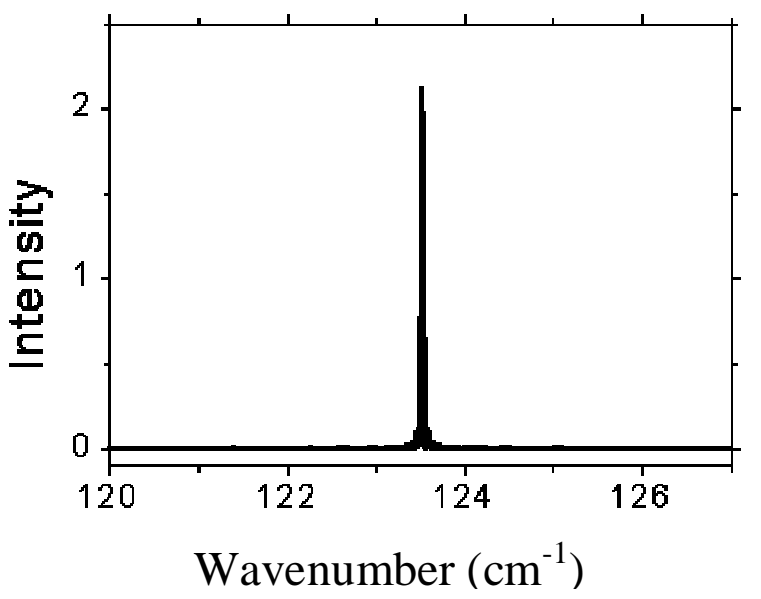

Figure 1 - Spectrum of p-Ge laser with intracavity wavelength selection. The p-Ge laser is tunable throughout the region 50 $140 \mathrm{~cm}^{-1}(70-200 \mu \mathrm{m}, 1.5-4.2 \mathrm{THz})$.

A closed cycle refrigerator allows greater flexibility in configuring the laser cavity than can be achieved within the tight cylindrical confines of a superconducting solenoid bore. In the closed cycle refrigerator, there is a large volume of evacuated space and the opportunity exists to operate components at temperatures above $4 \mathrm{~K}$. For example, tunable wavelength selectors involve moving parts that may perform poorly at low temperatures. 


\section{EXPERIMENTAL}

Figure 2 presents a schematic of the experimental setup. The closed cycle refrigerator system includes a Janis Research SHI-4-15 Optical Cryostat and a Lakeshore Model 331S PID Temperature controller. The cold surface of the cryostat is a 3" copper flange connected to the second stage of the cold head, which provides $1.5 \mathrm{~W}$ of cooling power at $4.2 \mathrm{~K}$. A stainless steel radiation shield which surrounds the second stage cold surface is attached to the first stage (45 W at 50 $\mathrm{K})$. The compressor is connected to the cold head by flexible gas lines and the temperature controller is connected through an instrumentation feedthrough on the cryostat. The temperature controller regulates two heating elements under the cold surface for temperature dependent measurements. The laser control electronics include the HV supply and thyratron pulser. The magnetic field was provided in Voigt configuration by a SmCo permanent magnet assembly [4] mounted on the cold surface. The far-IR radiation was measured by a Si bolometer mounted above the teflon or polyethylene window at the top of the vacuum shroud of the cryostat.

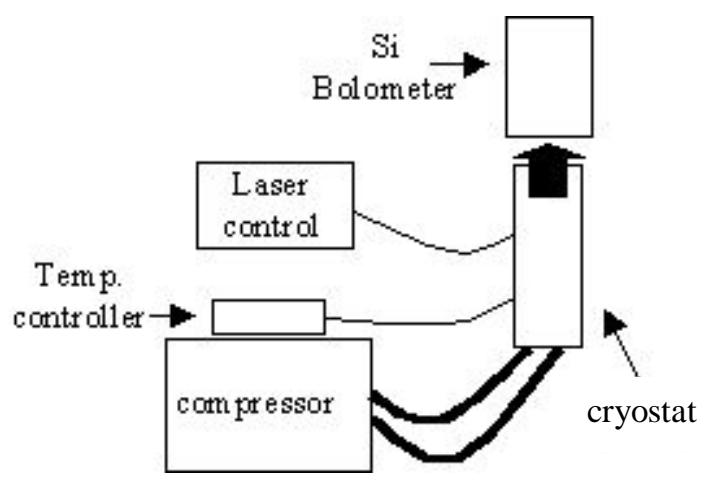

Figure 2 - Schematic of the closed cycle refrigerator system.

Figure 3 shows photographs of the control electronics (left) and the optical cryostat (right). The bottom portion of the 4 $\mathrm{K}$ cryostat that houses a down-looking silicon bolometer with $370 \mathrm{~cm}^{-1}$ low pass filter is visible resting on a platform above the cryostat. The outer vacuum shroud of the cryostat has a single teflon or polyethylene window on its end, through which the emitted far-IR radiation reaches the bolometer. The cryostat hangs from aluminum posts attached to the platform. 

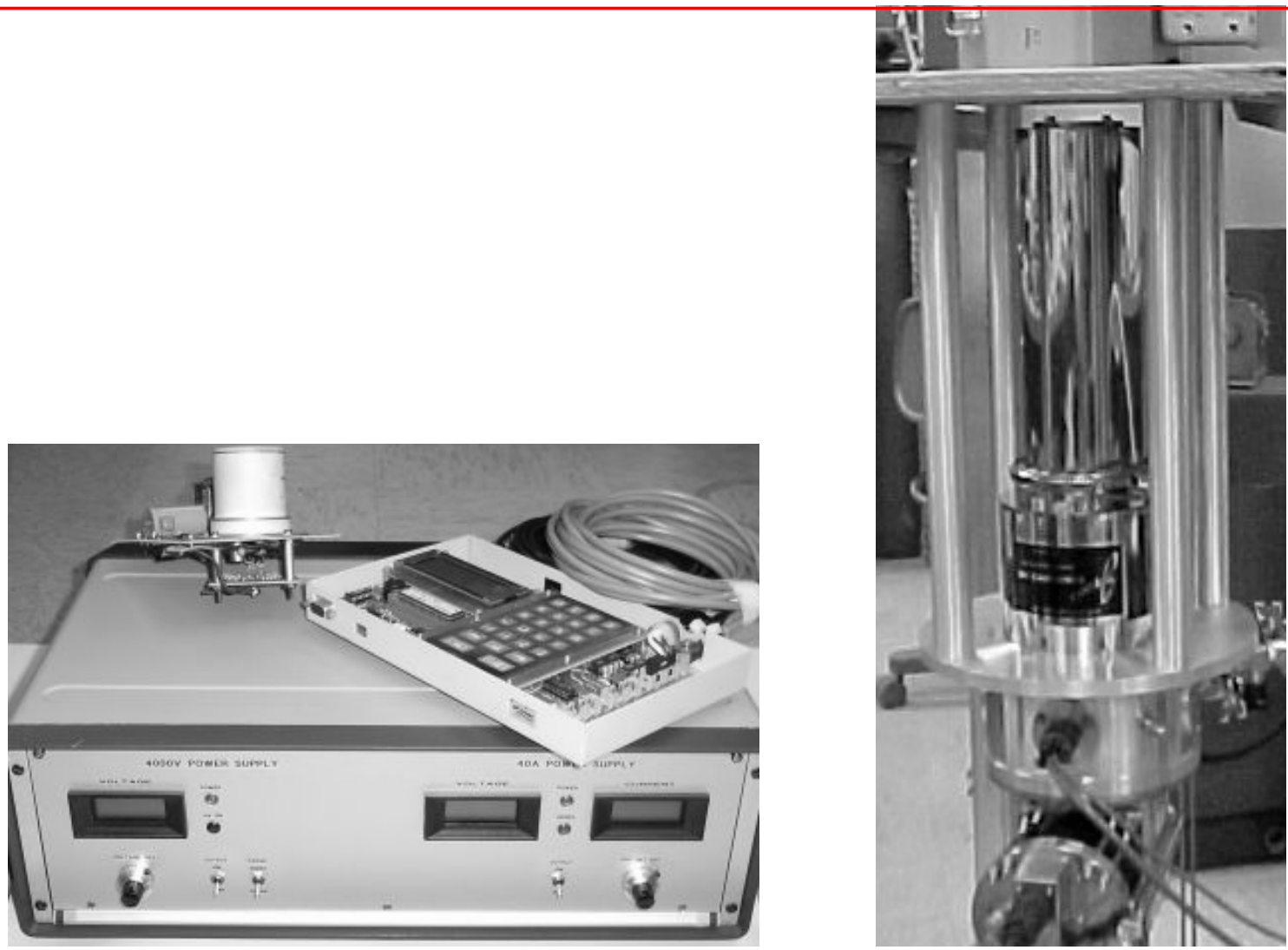

Figure 3 - Experimental setup. Compact custom laser control electronics (thyratron, pulse generator, and HV supply) are on the left. The case also includes a current supply to be used with traditional superconducting solenoids. The closed cycle cryostat is on the right.

A germanium laser rod was cut from gallium-doped single crystals to the shape of a rectangular parallelepiped with dimensions $51 \mathrm{~mm} \times 4.0 \mathrm{~mm} \times 3.45 \mathrm{~mm}$. Small end faces were polished flat and parallel to within 30 arcsec. Indium ohmic contacts were applied with an ultrasonic soldering iron to the narrow lateral faces. Copper mirrors were attached to the end faces as laser cavity mirrors.

Compact laser control electronics (Fig. 3) were developed by Zaubertek. These include a HV supply, a superconducting magnet supply (not used here), a compact thyratron switch for applying square high-power excitation pulses to the active crystal, and a keypad/computer - programmable trigger source to control the thyratron.

Fig. 4 (left) shows the cold surface of the closed cycle refrigerator. Fig. 4 (center) shows the SmCo permanent magnet assembly atop a copper flange that attaches to the cryostat cold surface. The flange is electrically isolated from the cold head by a silicon wafer. The top of the p-Ge crystal and copper output mirror are visible inside the magnet. A copper radiation shield (not shown) is mounted on the second stage, around the magnet. Fig. 4 (right) shows the laser crystal with copper mirrors attached, soldered with Indium to a copper cold finger which acts as the HV ground and provides thermal contact with the cold surface of the cryostat. The cold finger is tapered and attached only to the lowest few millimeters of the crystal to avoid breaking the crystal from differential thermal contraction. 


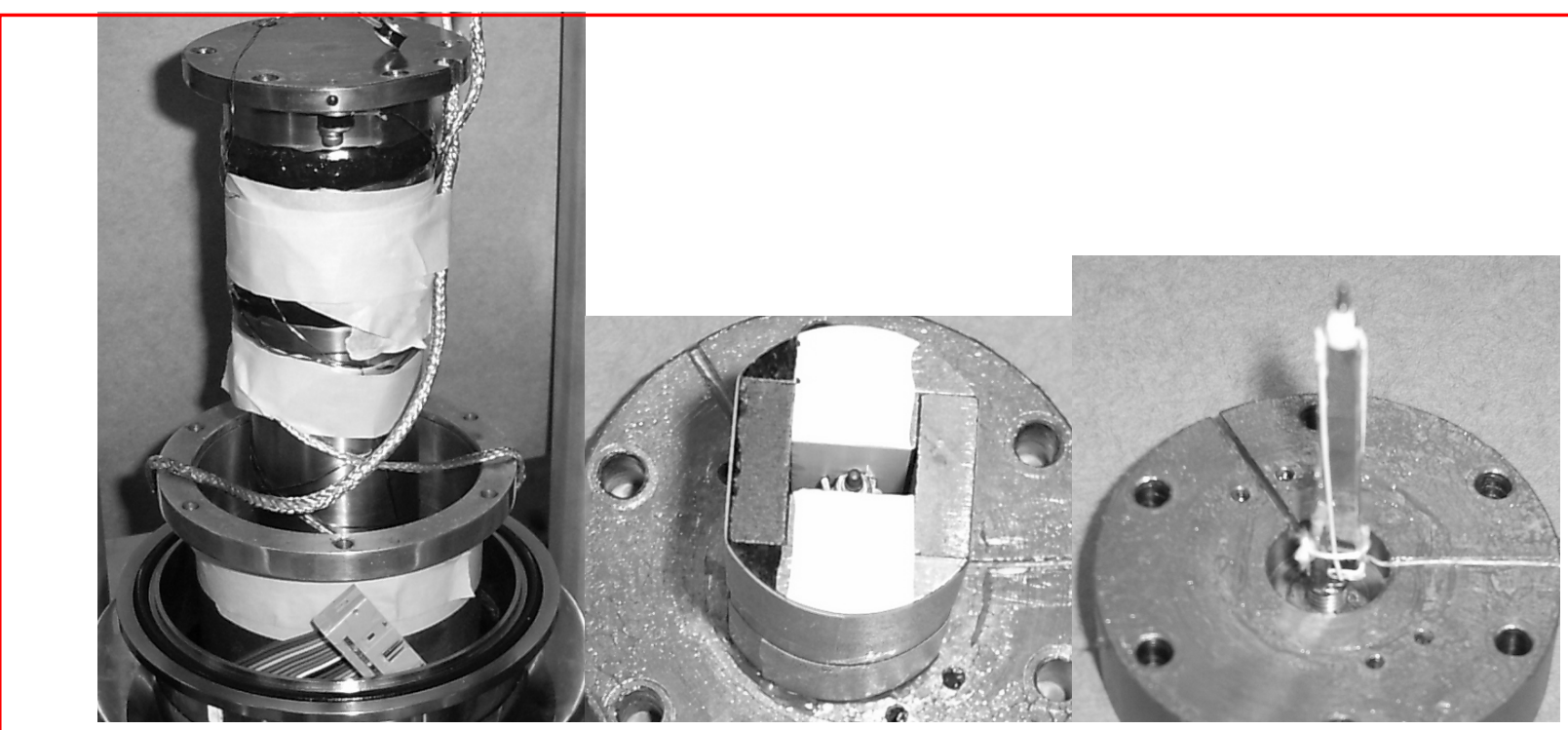

Figure 4 - Photographs of the cold head, permanent magnet, and p-Ge crystal mounted on cold finger.

Temperature of the cold surface is monitored by two GaAlAs diode temperature sensors. Both can be seen in Fig.4 (left). One is mounted to the underside of the cold surface and the other is typically mounted on the laser crystal cold finger, near the edge of the $\mathrm{p}-\mathrm{Ge}$ crystal.

\section{RESULTS}

Figure 5 presents laser emission intensity as a function of applied electric field for two different pulse durations. The 2 $\mu$ s curve jumps at $1.8 \mathrm{kV} / \mathrm{cm}$ to a regime of laser operation for Ga-doped Ge lasers known as the "high field" region. The high field signal is expected to be about 4-5 times stronger than the low field signal, when measured with a detector with flat response, like the bolometer used, and this is observed in Fig. 5. These data indicate that the applied magnetic field from the SmCo assembly is on the border between low and high field regions of operation.

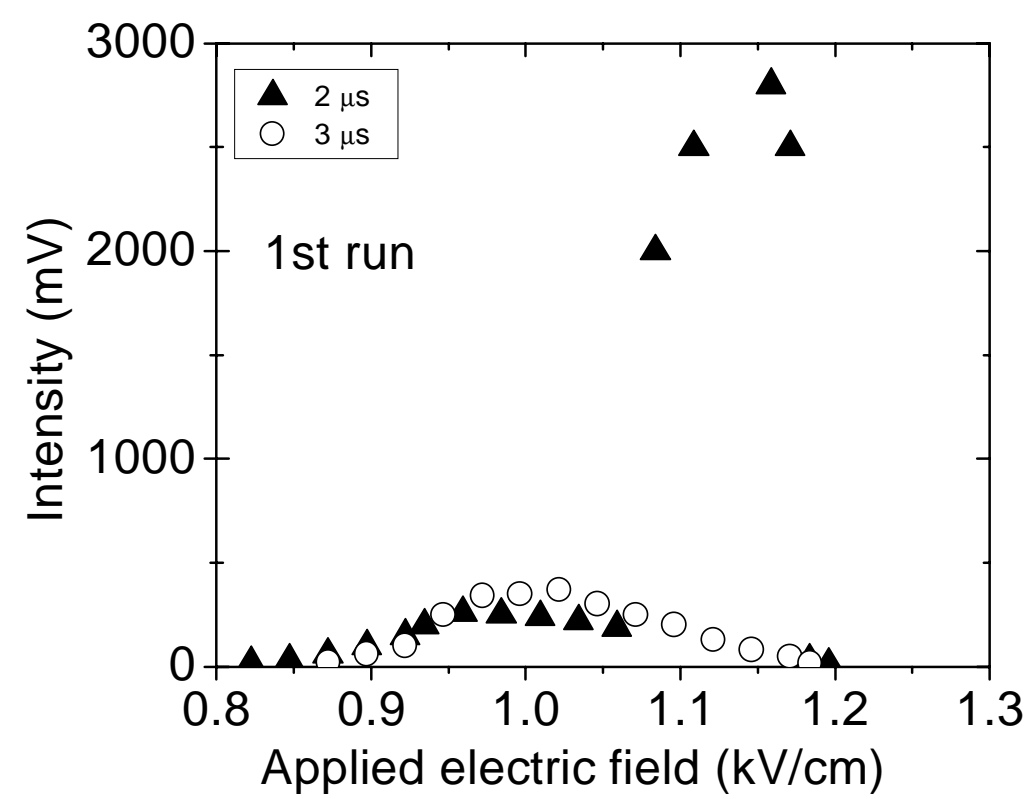

Figure 5 - Instability in power level. Indicates that B-field is at the border between low and high field zones. 


\begin{abstract}
The high-field region is favorable for broad wavelength tuning, mode-locking, and high output power. In order to reliably access the high field region, a new magnet has been designed. Figure 6 compares the calculated field strength and uniformity for the permanent magnet currently in use and the newly designed magnet. The magnet currently in use has a room temperature field in the air gap of $\sim 0.68 \mathrm{~T}$, while the new design should give a field of $\sim 1.02 \mathrm{~T}$. The fields of these magnets drop about $18 \%$ on cooling to $4 \mathrm{~K}$ [4]. The new design also has a much wider region of uniform field because of the use of iron pole pieces.
\end{abstract}
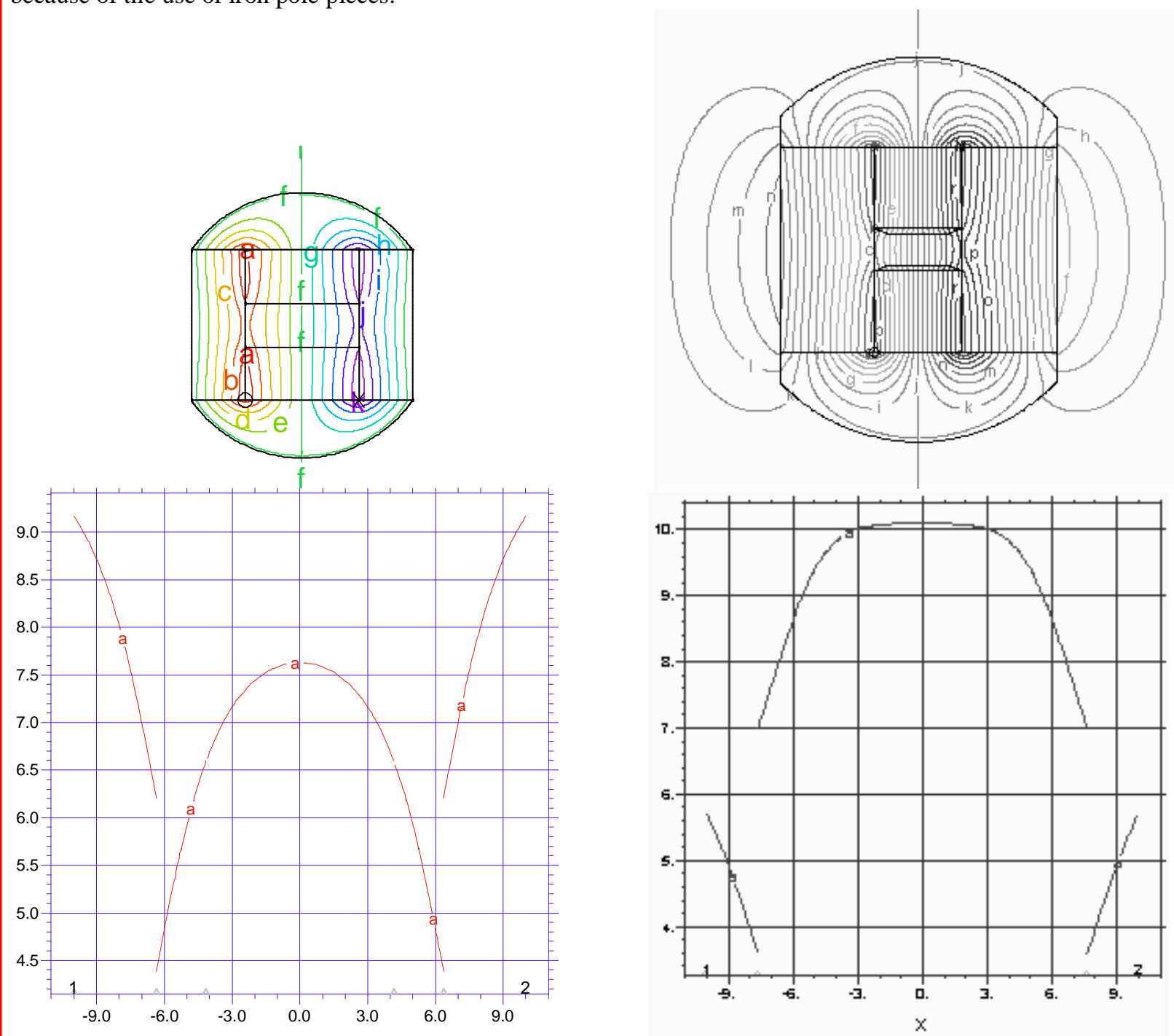

Figure 6 - Comparison of current (left) and new (right) SmCo magnet assembly designs. The upper figures show cross sections of the magnets with calculated magnetic field lines. The large square and rectangular elements are SmCo. The curved elements are iron yokes. The larger magnet has room for pole pieces. The central space is an air gap. The lower plots give horizontal cross sections of magnetic field strength $(\mathrm{kG})$ vs. distance from the center of the air gap in $\mathrm{mm}$. The magnet used for this paper (left) has a maximum field in the air gap of $0.68 \mathrm{~T}$. The new design (right) has a maximum field of 1.02T. Note the wider region of uniformity within the air gap for the new design. 
Ge laser operation has been obtained twice to date in our closed-cycle cryostat with the smaller magnet assembly. Each successful run was separated by about 1 month and the system was reassembled between runs. The next graphs compare results of the two runs.

Figure 7 shows the measured crystal resistance as a function of temperature in the range of temperatures where lasing was observed. The crystal resistance is a fixed function of its temperature. The crystal resistance vs. cold-head temperature curves are nearly the same in the two runs. This means that the cold surface temperature is a repeatable measure of the (relative) crystal temperature, within about 1 degree. Resistance increases with decreasing temperature because of carrier freeze-out.

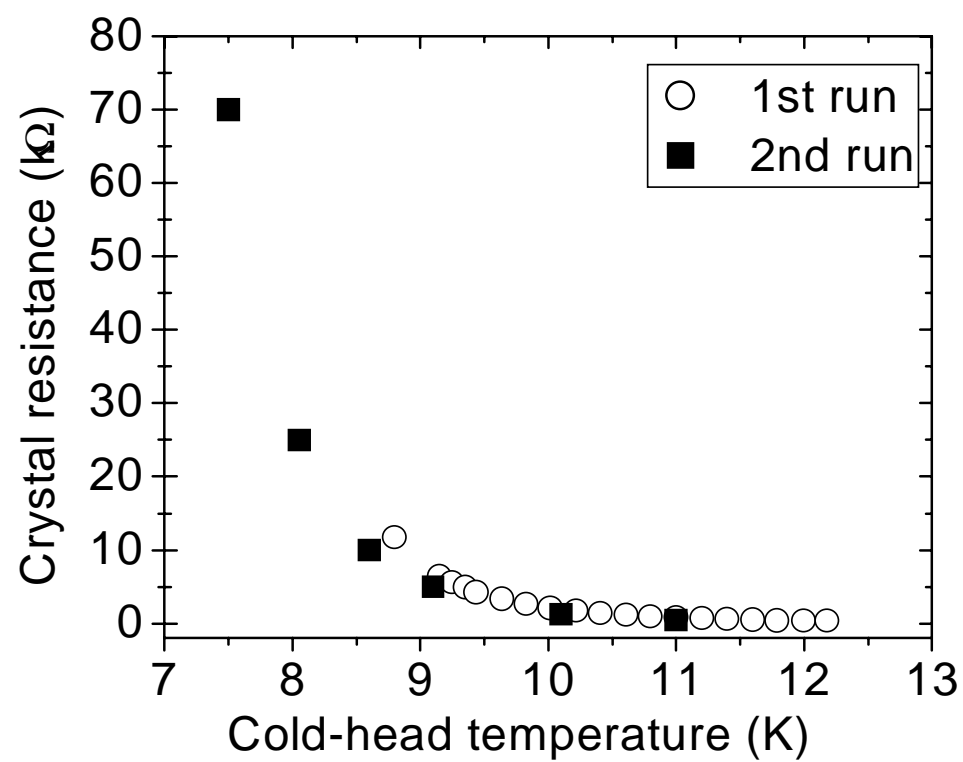

Figure 7 - Measured crystal resistance as a function of cold head temperature over the span of temperatures where lasing was observed.

The intensity of far-IR radiation measured with the Si bolometer as the cold head temperature was varied is shown in Fig. 8. Lower temperatures were reached in Run 2, but the laser seemed to require these lower temperatures to function.

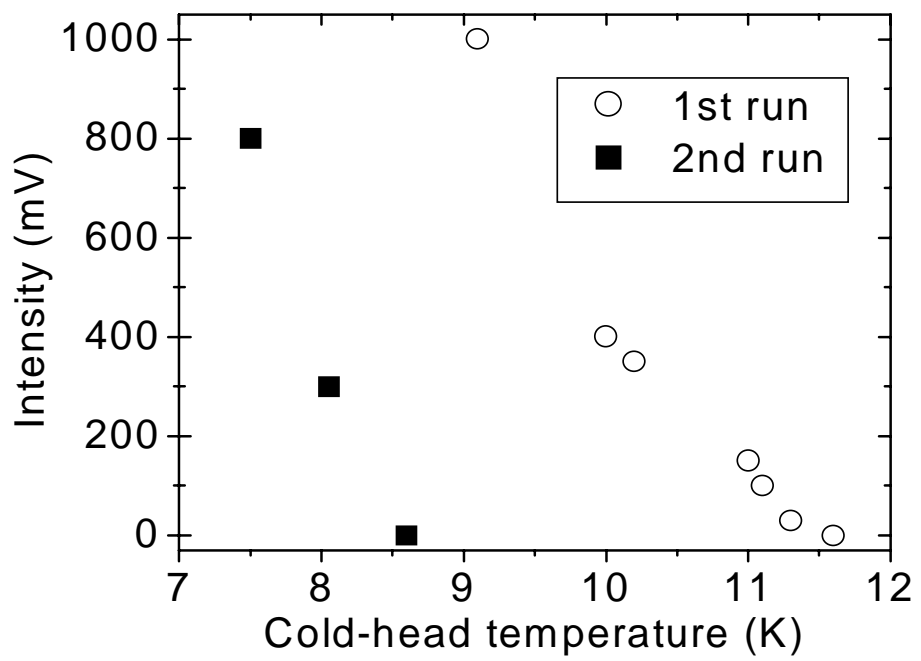

Figure 8 - Intensity of far-IR radiation detected by the Si bolometer as a function of cold-head temperature. 
Figure 9 shows the measured far-IR intensity as the electric field applied to the laser was varied. For these data, the laser was operating in the low-field mode. The range of electric fields at which lasing was observed was more narrow for Run 2.

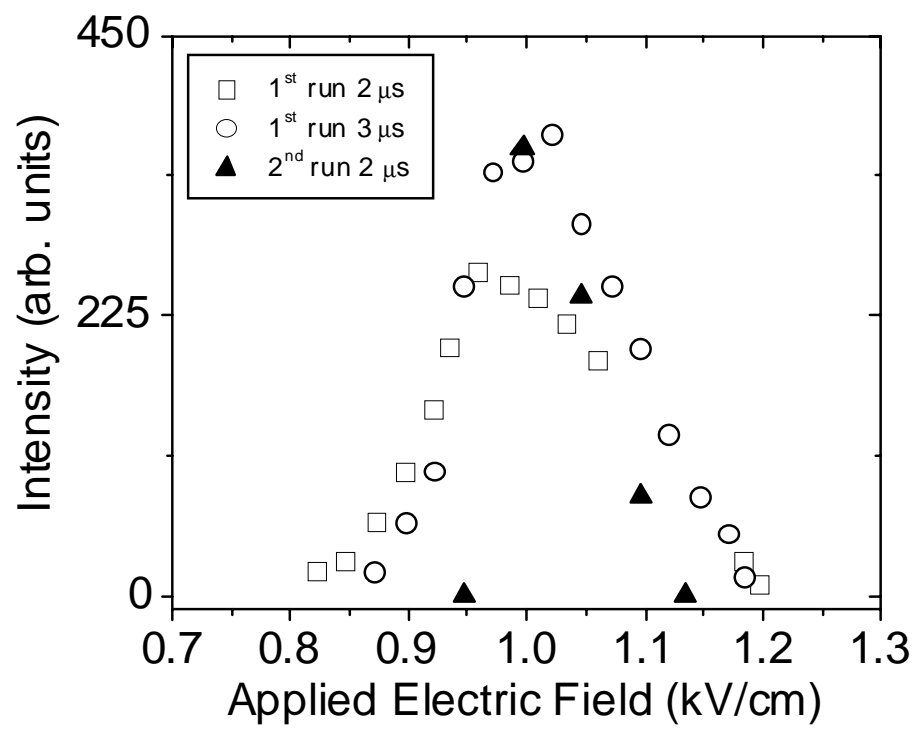

Figure 9 - Far-IR intensity vs. applied electric field for the two runs.

The measured current-voltage characteristics of the laser crystal are shown in Fig. 10 (curve). The current and voltage values when the laser was emitting radiation are indicated in the plot by symbols, which for both runs fall along the independently measured curve.

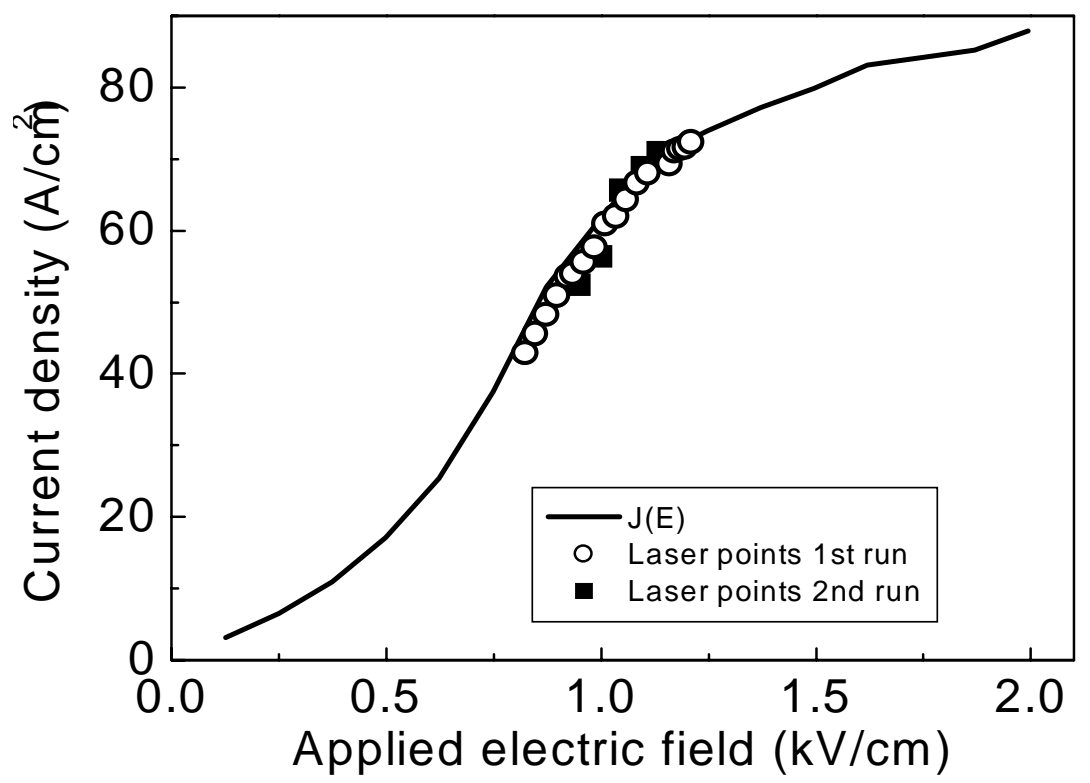

Figure 10 - The solid curve is from the measured current-voltage relationship. The shape of the curve is characteristic of Voigt geometry with current saturation at highest fields. The symbols indicate where lasing was observed in the two runs.

Figure 11 shows results of varying the duration of the HV pulse applied to the laser for different fixed repetition rates. If the pulse duration is too short $(<1.5$ microsecond), the laser doesn't lase or the emission pulse is less than its natural 
duration, resulting in no or low signal at the slow bolometer. When the pulse is long, the average applied power is high, eventually overcoming the ability of the cooler to remove heat from the active crystal.
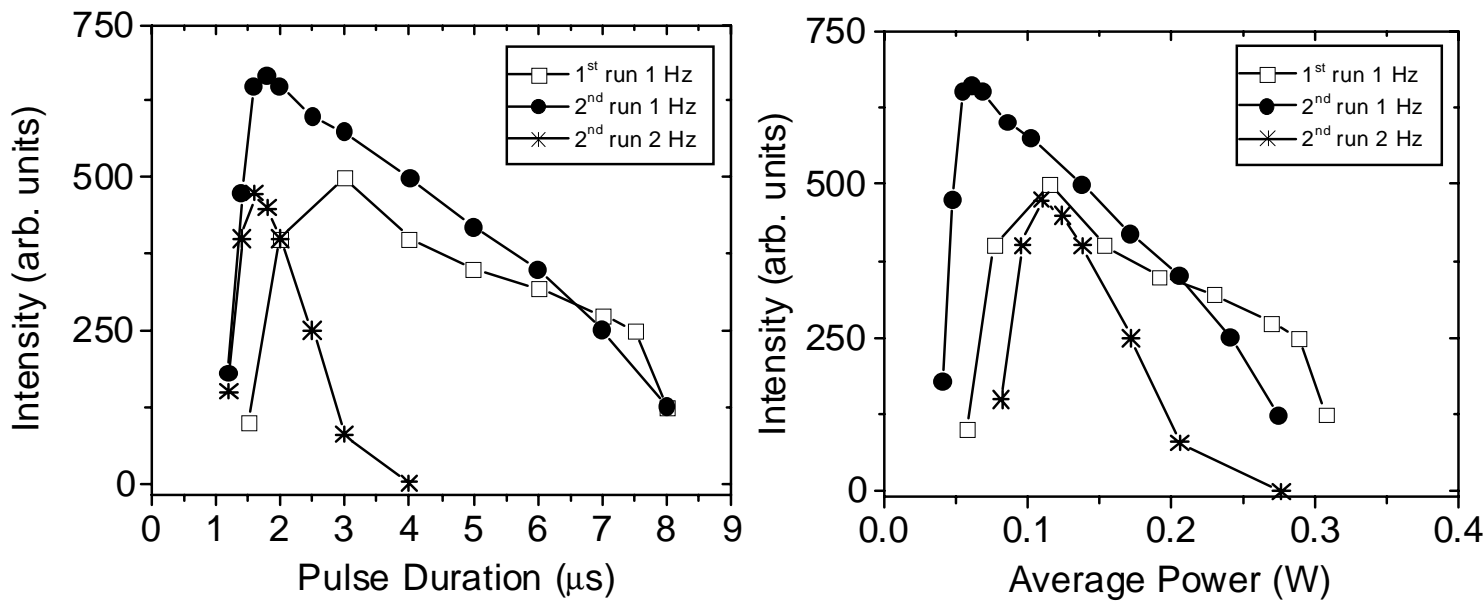

Fig. 11 - Intensity plotted against pulse duration (left) and average power (right) for repetition rates of 1 and $2 \mathrm{~Hz}$.

Figure 12 shows results of varying the repetition rate of the applied HV pulses for different fixed pulse durations. Even though the crystal reached a lower temperature on the second run (Fig. 8), heat extraction appears to be worse for this run.
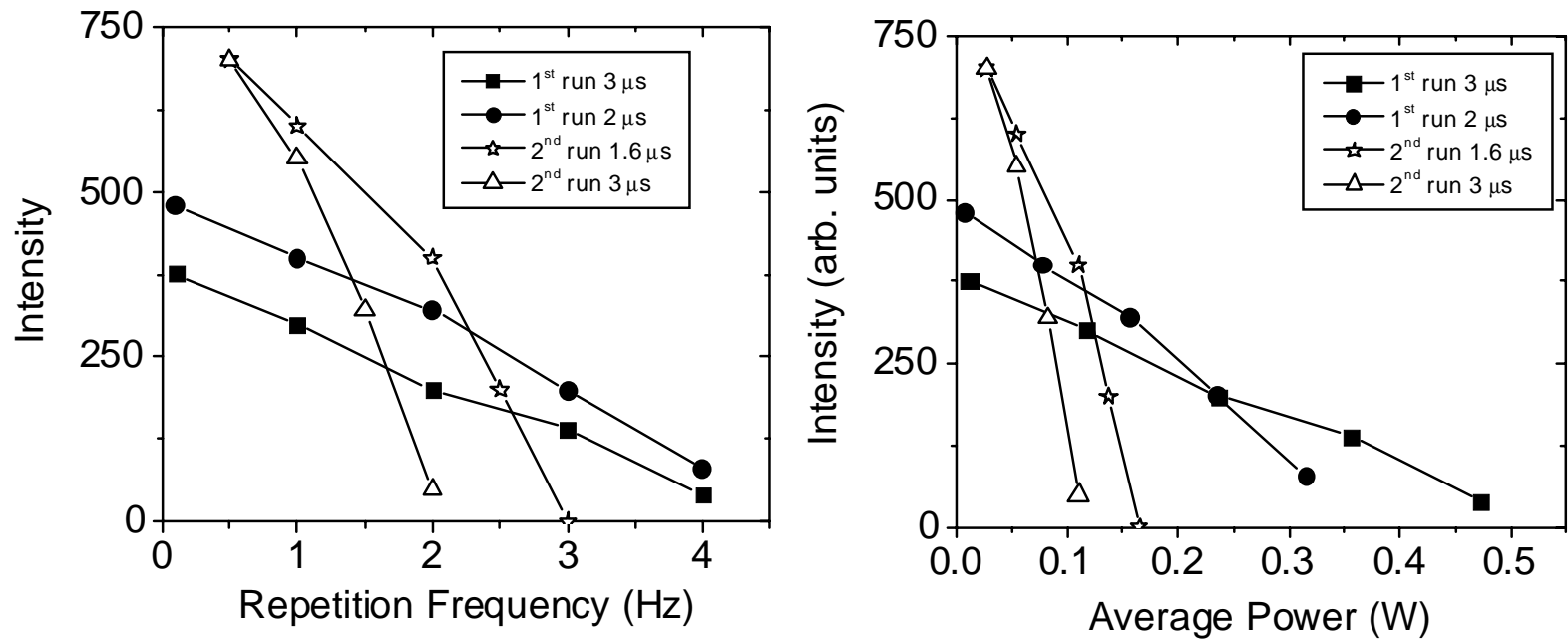

Figure 12 - Intensity plotted against repetition frequency (left) and average power (right) for different fixed pulse durations.

\section{DISCUSSION and CONCLUSION}

Fig. 6 shows that the small SmCo assembly used to produce the results in this paper has a field that is too low to be generally useful, since not all Ga-doped Ge laser rods are of sufficient quality to operate in the low-field zone. Moreover, the rather low uniformity of the field makes placement of the crystal in the magnet rather critical. This fact, combined with poor control over field angle may explain differences in performance between runs 1 and 2 . The new magnet should alleviate these problems.

These data indicate that it is possible to remove up to $0.5 \mathrm{~W}$ while keeping the crystal temperature sufficiently low to produce lasing. The cold head is rated for $1.5 \mathrm{~W}$ at $4 \mathrm{~K}$, which means that the duty cycle can be improved at most by a factor 3 for the laser crystal used. This can be achieved through better thermal coupling. There are several potential alternative configurations in development. 
In conclusion, we have achieved operation of a bulk p-Ge laser in a closed cycle refrigerator with a SmCo permanent magnet assembly. Continued development of this turn-key system will make the $\mathrm{p}$-Ge laser accessible for applications. The results presented will be used to guide the continued development of the system.

\section{ACKNOWLEDGEMENTS}

This work was supported by AFOSR contracts F49620-C-02-0027 and F49620-01-1-0339.

\section{REFERENCES}

1. E. Bründermann, "Widely Tunable Far Infrared Hot Hole Semiconductor Lasers," in Long-Wavelength Infrared Semiconductor Lasers, Editor: H. Choi (John Wiley and Sons, New York, 2004) Chapter 6.

2. A. V. Muravjov, S. H. Withers, H. Weidner, R. C. Strijbos, S. G. Pavlov, V. N. Shastin, and R. E. Peale, "Single axial-mode selection in a far-infrared p-Ge laser," Appl. Phys. Lett. 76, 1996 (2000).

3. Kijun Park, R. E. Peale, H. Weidner, and J. J. Kim, "Submillimeter p-Ge laser using a Voigt-configured permanent magnet," IEEE J. Quantum Electronics 32, 1203 (1996).

4. C. J. Fredricksen, E. W. Nelson, A. V. Muarvjov, and R. E. Peale, "High field p-Ge laser operation in permanent magnet assembly," Infrared Physics \& Technology, 44, 79 (2003).

5. E. Brundermann, H.P. Roser, "First Operation of a far-infrared p-germanium laser in a standard closed-cycle machine at 15 K," Infrared Physics and Technology, 38, 201 (1997). 